Supporting Information for

\title{
Structure of a uranyl peroxo complex in aqueous solution from first-principles molecular dynamics simulations.
}

\author{
Michael Bühl, Nicolas Sieffert and Georges Wipff
}

\section{Additional Computational Details}

\section{CPMD simulations}

The same methods and basis sets as in our previous studies of uranyl complexes ${ }^{1}$ were employed. Car-Parrinello molecular dynamics (CPMD) ${ }^{2}$ simulations were performed using the BLYP functional ${ }^{3}$ and norm-conserving pseudopotentials that had been generated according to the Troullier and Martins procedure ${ }^{4}$ and transformed into the Kleinman-Bylander form. ${ }^{5}$ For uranium, the semicore (or small-core) pseudopotential was employed that had been generated and validated in reference 6 . Periodic boundary conditions were imposed using cubic supercells with a lattice contant of $13 \AA$. Kohn-Sham orbitals were expanded in plane waves at the $\Gamma$-point up to a kinetic energy cutoff of $80 \mathrm{Ry}$. Simulations were performed in the NVT ensemble using a single NoséHoover thermostat set to $320 \mathrm{~K}$ (frequency $1800 \mathrm{~cm}^{-1}$ ), a fictitious electronic mass of 600 a.u., and a time step of $0.121 \mathrm{fs}$. The boxes contained uranyl, one peroxo and one hydroxo ligand, and a total of 57 water molecules, affording a density of ca. 1.0. The system has one negative charge, neutralised by a uniform background charge. In order to maintain the time step, hydrogen was substituted with deuterium. Long-range electrostatic interactions were treated with the Ewald method. No electrostatic decoupling between replicated cells was included.

Constrained CPMD simulations were performed along a predefined reaction coordinate $\xi$ (difference in bond distances $\Delta r$ ) connecting the different tautomeric complexes, in order to evaluate the change in the Helmholtz free energy by pointwise thermodynamic integration ${ }^{7}$ of the mean constraint force $\langle f\rangle$ along this coordinate via

$$
\Delta A_{\mathrm{a} \rightarrow \mathrm{b}}=-\int_{\mathrm{a}}^{\mathrm{b}}\langle f(\xi)\rangle \mathrm{d} \xi
$$

At each point, the system was propagated until $\langle f(\xi)\rangle$ was sufficiently converged (usually within 1.5 - 2 ps after 0.5 ps of equilibration, similar to the degree of convergence documented in Figure S1 of the supporting information for reference 6 . Each new point was continued from the final, equilibrated configuration of the previous one, using 3200 steps of continuous slow growth to decrease the constrained $\Delta r$. The point with largest $\langle f(\xi)\rangle$, at $\Delta r=0.31 \AA$, was retraced with a higher cutoff of $100 \mathrm{Ry}$ and a timestep of 3 a.u. (for a total of 3 ps, restarted from the last point of the slow growth at $80 \mathrm{Ry}$ after quenching to the Born-Oppenheimer surface). According to the root-meansquare displacement along the trajectories, the solutions remained liquid-like throughout. 
Selected conformers were optimised in the gas phase until the maximum gradient was less than $5 \cdot 10^{-4}$ a.u. (denoted CP-opt).

\section{QM/MM simulations:}

We employed the CPMD/MM protocol previously validated, ${ }^{8}$ where the solute (a single complex 1a or 2a) is described at the same DFT level, whereas the solvent is described by the TIP3P model. ${ }^{9}$

The solvent consists of $3000 \mathrm{H}_{2} \mathrm{O}$ molecules in a periodic box of cell length $45.05 \AA$. Van-derWaals parameters on $\mathrm{OH}^{-}, \mathrm{H}_{2} \mathrm{O}, \mathrm{O}_{2}{ }^{2-}$ and $\mathrm{OOH}^{-}$ligands were the same as in the TIP3P model. ${ }^{9}$ The systems were pre-equilibrated using the following sequence:

(i) Classical pre-equilibration: After 1000 steps of energy minimisation, 100 ps of MD were performed in the NVT ensemble ( $\mathrm{T}=320 \mathrm{~K}$, starting from random velocities), followed by $100 \mathrm{ps}$ of MD in the NPT ensemble $(\mathrm{P}=1 \mathrm{~atm}, \mathrm{~T}=320 \mathrm{~K})$ and $500 \mathrm{ps}$ of $\mathrm{MD}$ in the NVT ensemble $(\mathrm{T}=$ $320 \mathrm{~K})$. These simulations were performed with the AMBER10 software. ${ }^{10}$ During the fully classical MD simulations, the solute was kept frozen ("belly" option in AMBER). Atomic charges on 1a and 2a were RESP charges computed at the B3LYP/6-31G** level and using a hyperbolic restraint of 0.001 a.u. (computed with the NWChem 6.1.1 software). ${ }^{11}$ Atomic charges along with the corresponding cartesian coordinates are gathered below:

13

XYZ coordinates of $\mathbf{1 a}+$ RESP charges (hyperbolic restraint 0.001 u.a.)

U $\quad 0.000360 \quad-0.109678 \quad-0.004974 \quad 2.2711$

$\begin{array}{llllll}\text { O } & 0.420415 & -0.229290 & -1.774084 & -0.7636\end{array}$

$\begin{array}{llllll}\text { O } & -0.159829 & 0.306709 & 1.771761 & -0.7855\end{array}$

$\begin{array}{llllll}\mathrm{O} & -2.193063 & -0.216715 & -0.343090 & -0.4879\end{array}$

$\begin{array}{llllll}\text { O } & -1.667189 & -1.517307 & -0.020266 & -0.5328\end{array}$

$\begin{array}{llllll}\text { O } & 1.964611 & -1.203093 & 0.514618 & -0.9441\end{array}$

$\begin{array}{llllll}\text { O } & 2.397315 & 1.277379 & -0.265787 & -0.8133\end{array}$

$\begin{array}{llllll}\mathrm{O} & -1.263648 & 2.299932 & 0.026087 & -0.7488\end{array}$

$\begin{array}{lllll}\mathrm{H} & 2.093548 & -1.395682 & 1.449362 & 0.3207\end{array}$

$\begin{array}{lllll}\mathrm{H} & 2.419933 & 1.241774 & -1.229313 & 0.3716\end{array}$

$\begin{array}{lllll}\mathrm{H} & 2.639435 & 0.367036 & 0.046293 & 0.4021\end{array}$

$\begin{array}{lllll}\mathrm{H} & -2.060740 & 1.747836 & -0.059309 & 0.3416\end{array}$

$\begin{array}{lllll}\mathrm{H} & -1.114191 & 2.388472 & 0.976634 & 0.3689\end{array}$
13

XYZ coordinates of $\mathbf{2 a}+$ RESP charges (hyperbolic restraint 0.001 u.a.)

$\begin{array}{lllll}\text { U } & 0.007416 & 0.023985 & 0.041886 & 2.4101\end{array}$

$\begin{array}{lllll}\mathrm{O} & -0.057091 & 0.294623 & 1.822235 & -0.7196\end{array}$

O $\quad 0.009075 \quad-0.189353-1.760825 \quad-0.7664$

$\begin{array}{llllll}\text { O } & 2.509185 & 0.148509 & -0.180010 & -0.4808\end{array}$

$\begin{array}{llllll}\text { O } & 2.001400 & -1.192941 & 0.151702 & -0.5349\end{array}$

$\begin{array}{llllll}\text { O } & -1.331436 & -1.879152 & 0.176594 & -0.9180\end{array}$

$\begin{array}{llllll}\text { O } & -2.672118 & 0.297489 & -0.144954 & -0.7439\end{array}$

$\begin{array}{llllll}\mathrm{O} & -0.044555 & 2.219274 & -0.258064 & -0.9480\end{array}$

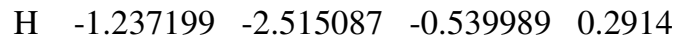

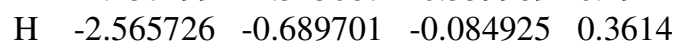

$\begin{array}{lllll}\mathrm{H} & -2.810539 & 0.533570 & -1.068094 & 0.3381\end{array}$

$\begin{array}{lllll}\mathrm{H} & -0.079572 & 2.792910 & 0.514311 & 0.3281\end{array}$

$\begin{array}{lllll}\mathrm{H} & 2.695045 & 0.084063 & -1.128228 & 0.3825\end{array}$

(ii) DFT/MM pre-equilibration: The last positions from the classical pre-equilibration step were taken and used as starting coordinates for an additional 3 ps of MD in the NVT ensemble $(\mathrm{T}=320$ $\mathrm{K}$ ) starting from random velocities, using the CPMD/Gromos96 interface of CPMD. ${ }^{12}$ The exact same simulation parameters as for full CPMD simulations were employed to describe the QM region. A non-periodic QM box of cell-length $15 \AA$ was employed and the periodic images were decoupled using the Martyna-Tuckerman approach. ${ }^{13}$ The so-called "NN" and "ESP" domains were defined by a $16 \AA$ and $36 \AA$ cutoff, respectively. Typically, 200 and 1200 water molecules were included in the "NN" and "ESP" regions, respectively. Built-in values for the covalent radii $\left(r_{c, j}\right)$ of MM atoms were used. ${ }^{12}$ Wannier function centers (WFCs) ${ }^{14}$ were computed on the fly every 50 timesteps during production runs of $15 \mathrm{ps}$ (NVT, at $320 \mathrm{~K}$ ).

Full citations of the Gaussian suite of programs are provided in reference 15. 
a) Gas phase
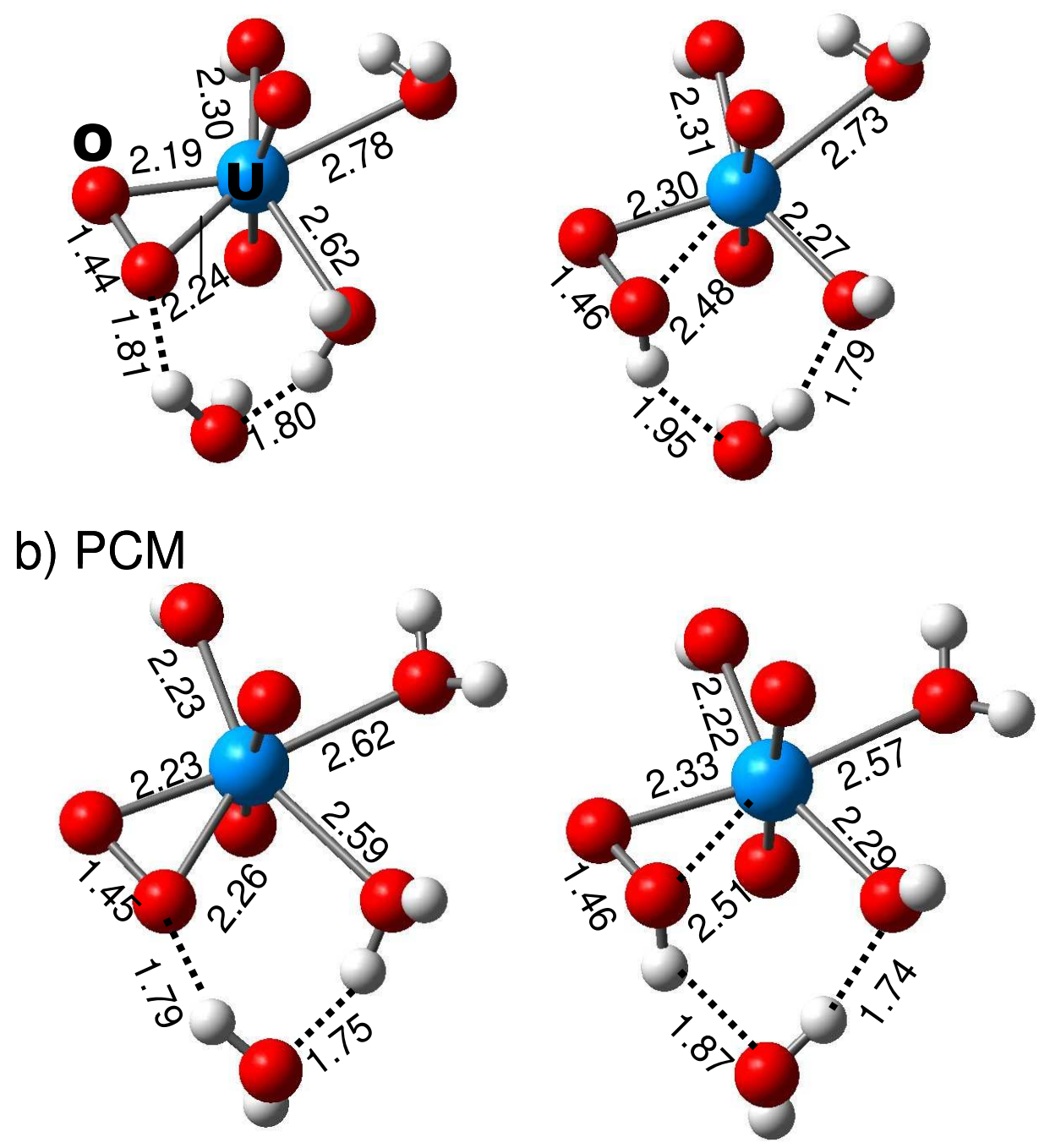

$1 \mathrm{a} \cdot \mathrm{H}_{2} \mathrm{O}$

$2 \mathrm{a} \cdot \mathrm{H}_{2} \mathrm{O}$

Figure S1: B3LYP/SDD optimised structures of $\mathbf{1 a} \cdot \mathrm{H}_{2} \mathrm{O}$ and $\mathbf{2 a} \cdot \mathrm{H}_{2} \mathrm{O}$ in a) the gas phase, and b) a continuum (PCM); selected distances are given in $\AA$. Note the intramolecular H-bond between the water ligand trans to the peroxo moiety and the adjacent $\mathrm{OH}$ group that is present in the gas phase (top), but absent in solution (bottom). 


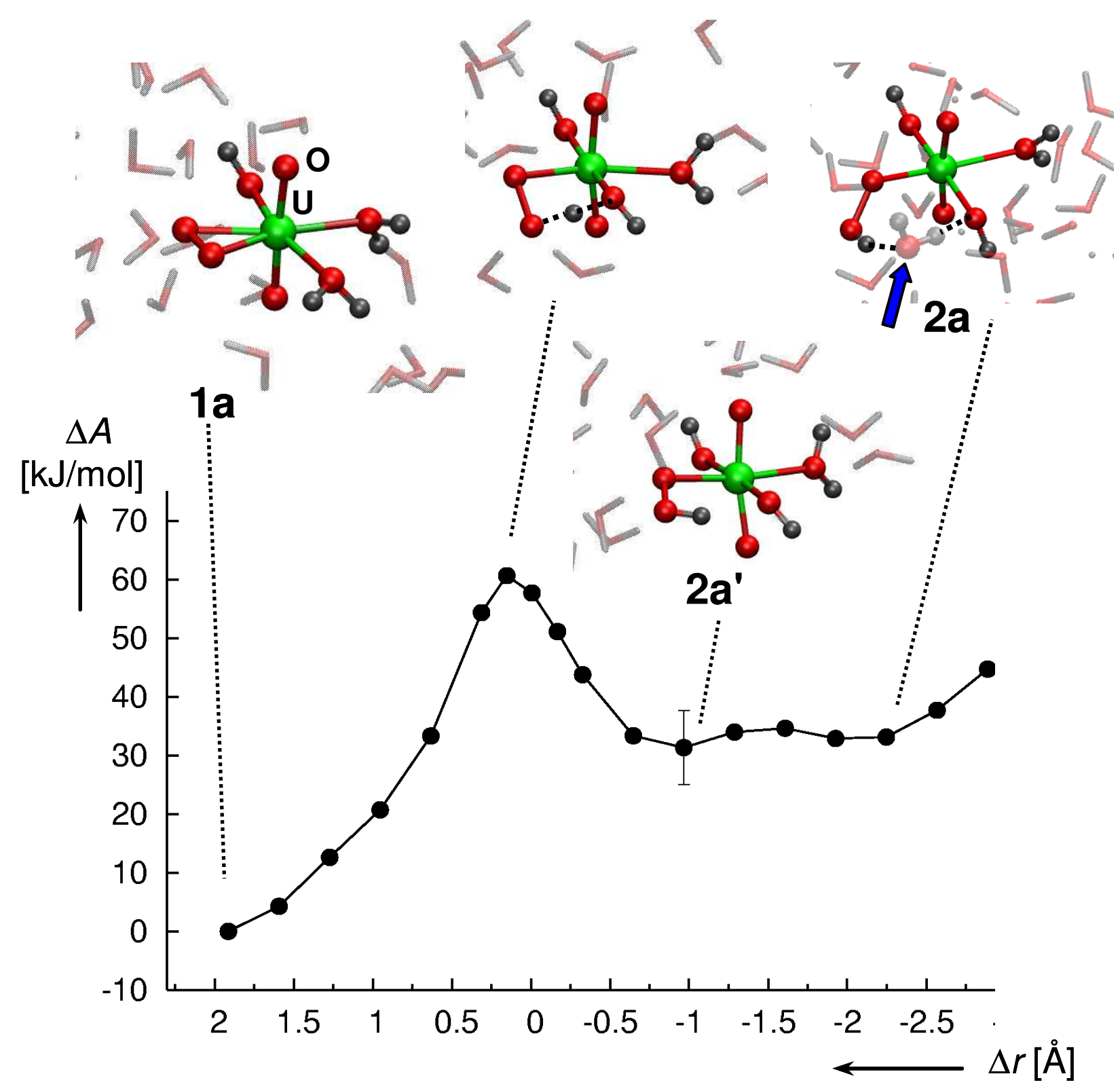

Figure S2: Magnified Figure 2 of the main paper. The water molecule from the solvent interacting with the $\mathrm{OH}$ groups from the complex in $\mathbf{2 a}$ is highlighted with a blue arrow. 

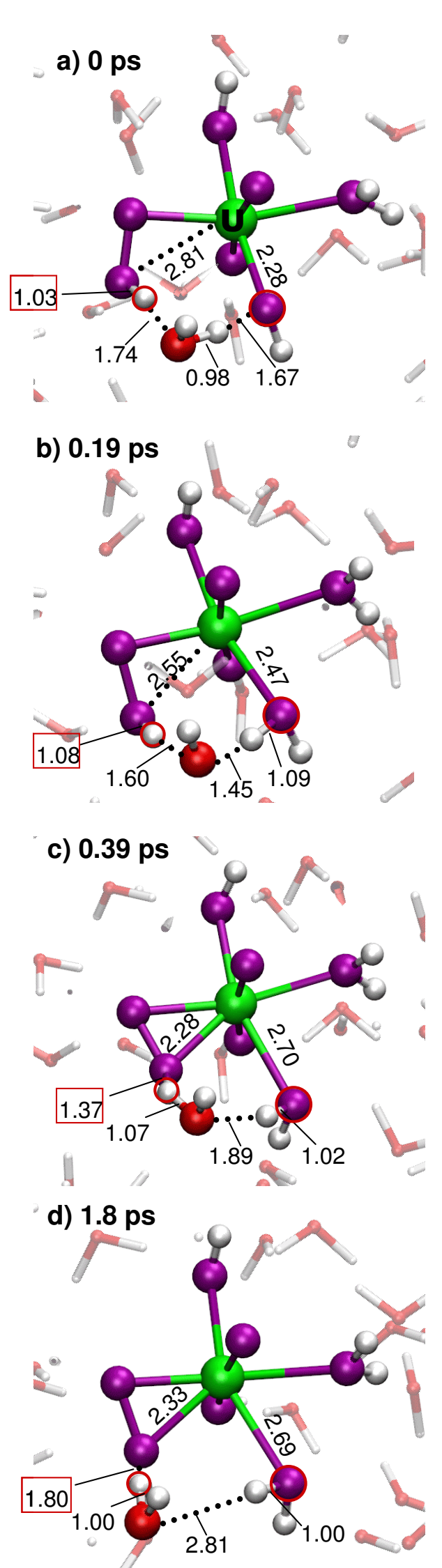

Figure S3: Spontaneous proton transfer during the constrained PTI step at $\Delta r=-1.61 \AA$; the OH distance in the red box is included in the constraint, as well as the $\mathrm{O} \cdots \mathrm{H}$ distance between the atoms in red circles. Note how on going from the instantaneous snapshots a) to d) the solvent molecule (O atom shown in red) acts as relay for proton transfer from the $\mathrm{OOH}$ to the $\mathrm{OH}$ ligand. $\mathrm{O}$ atoms of the complex are shown in purple, selected distances are given in $\AA$. 
S6

Table S1: Average (and standard deviation in parenthesis) of energy components from the PCM model $;^{a} 50$ single points from the constrained CPMD simulations.

\begin{tabular}{|c|c|c|}
\hline Energy term & $1 \mathbf{a}$ & $2 \mathbf{a}$ \\
\hline \multicolumn{3}{|c|}{ Gas phase } \\
\hline SCF energies (in Hartree) & $-1006.78611(864)$ & $-1006.79774(870)$ \\
\hline \multicolumn{3}{|c|}{ In solution $(P C M)$} \\
\hline SCF energies (in Hartree) & $-1006.95124(467)$ & $-1006.93698(579)$ \\
\hline Total free energy in solution (in Hartree) ${ }^{b}$ & $-1006.93351(461)$ & $-1006.91853(571)$ \\
\hline Polarized solute - solvent $(\mathrm{kcal} / \mathrm{mol})$ & $-117.0(45)$ & $-97.6(41)$ \\
\hline Cavitation energy ( $\mathrm{kcal} / \mathrm{mol})$ & $21.9(26)$ & $22.3(28)$ \\
\hline Dispersion energy (kcal/mol) & $-12.3(1)$ & $-12.2(1)$ \\
\hline Repulsion energy (kcal/mol) & $1.5(1)$ & $1.5(1)$ \\
\hline Total non electrostatic (kcal/mol) & $11.1(27)$ & $11.6(28)$ \\
\hline
\end{tabular}

${ }^{a}$ Gaussian03 settings, BLYP/SDD/6-311+G** level; only the pristine complexes 1a or 2a were considered (the solvent has been removed at each step). ${ }^{b}$ Including all non electrostatic terms.

\section{References}

(1) Bühl, M.; Sieffert, N.; Wipff, G. Chem. Phys. Lett. 2009, 467, 287-293.

(2) Car, R.; Parrinello, M. Phys. Rev. Lett. 1985, 55, 2471-2474.

(3) (a) Becke, A. D. Phys. Rev. A 1988, 38, 3098-3100. (b) Lee, C.; Yang, W.; Parr, R. G. Phys. Rev. B 1988, 37, 785-789.

(4) Troullier, N.; Martins, J. L. Phys. Rev. B 1991, 43, 1993-2006.

(5) Kleinman, L.; Bylander, D. M. Phys. Rev. Lett. 1982, 48, 1425-1428.

(6) Bühl, M.; Diss, R.; Wipff, G. J. Am. Chem. Soc. 2005, 127, 13506-13507.

(7) Sprik, M.; Ciccotti, G. J. Chem. Phys. 1998, 109, 7737-7744, and references cited therein.

(8) Sieffert, N.; Bühl, M.; Gaigeot, M.-P.; Morrison, C. A. J. Chem. Theory Comput. 2013, 9, 106.

(9) Jorgensen, W. L.; Chandrasekhar, J.; Madura, J. D.; Impey, R. W.; Klein, M. L. J. Chem. Phys. 1983, 79, 926.

(10) D.A. Case, T.A. Darden, T.E. Cheatham, III, C.L. Simmerling, J. Wang, R.E. Duke, R. Luo, M. Crowley, R.C. Walker, W. Zhang, K.M. Merz, B. Wang, S. Hayik, A. Roitberg, G. Seabra, I. Kolossváry, K.F. Wong, F. Paesani, J. Vanicek, X. Wu, S.R. Brozell, T. Steinbrecher, H. Gohlke, L. Yang, C. Tan, J. Mongan, V. Hornak, G. Cui, D.H. Mathews, M.G. Seetin, C. Sagui, V. Babin, and P.A. Kollman (2008), AMBER 10, University of California, San Francisco.

(11) (a) Straatsma, T.P.; Aprà, E.; Windus, T.L.; Bylaska, E.J.; de Jong, W.; Hirata, S.; Valiev, M.; Hackler, M.; Pollack, L.; Harrison, R.; Dupuis, M.; Smith, D.M.A; Nieplocha, J.; Tipparaju V.; Krishnan, M.; Auer, A.A.; Brown, E.; Cisneros, G.; Fann, G.; Früchtl, H.; Garza, J.; Hirao, K.; Kendall, R.; Nichols, J.; Tsemekhman, K.; Wolinski, K.; Anchell, J.; Bernholdt, D.; Borowski, P.; Clark, T.; Clerc, D.; Dachsel, H.; Deegan, M.; Dyall, K.; Elwood, D.; Glendening, E.; Gutowski, M.; Hess, A.; Jaffe, J.; Johnson, B.; Ju, J.; Kobayashi, R.; Kutteh, R.; Lin, Z.; Littlefield, R.; Long, X.; Meng, B.; Nakajima, T.; Niu, S.; Rosing, M.; Sandrone, G.; Stave, M.; Taylor, H.; Thomas, G.; 
van Lenthe, J.; Wong, A.; Zhang, Z.; NWChem, A Computational Chemistry Package for Parallel Computers, Version 4.6 (2004), Pacific Northwest National Laboratory, Richland, Washington 99352-0999, USA. (b) Kendall, R.A.; Aprà, E.; Bernholdt, D.E.; Bylaska, E.J.; Dupuis, M.; Fann, G.I.; Harrison, R.J.; Ju,J.; Nichols, J.A.; Nieplocha, J.; Straatsma, T.P.;Windus, T.L.;Wong, A.T. Computer Phys. Comm., 2000, 128, 260-283; $\quad$ (c) Valiev, M.; Bylaska, E. J.; Govind, N.; Kowalski, K.; Straatsma, T. P.; Van Dam, H. J. J.; Wang, D.; Nieplocha, J.; Apra, E.; Windus, T. L.; de Jong, W. A. Comput. Phys. Commun. 2010, 181, 1477.

(12) Laio, A.; VandeVondele, J.; Rothlisberger, U. J. Chem. Phys. 2002, 116, 6941; (b) Laio, A.; VandeVondele, J.; Rothlisberger, U. J. Phys. Chem. B 2002, 106, 7300.

(13) Martyna, G. J.; Tuckerman, M. E. J. Chem. Phys. 1999, 110, 2810.

(14) (a) Marzari, N.; Vanderbilt, D. Phys. Rev. B 1997, 56, 12847; (b) Silvestrelli, P. L.; Marrzari, N.; Vanderbilt, D.; Parrinello, M. Solid State Commun. 1998, 107, 7. Wannier functions are a generalization to infinite periodic systems of the Boys localized orbitals: (c) Boys, S. F.; in Quantum Theory of Atoms, Molecules, and the Solid State (Löwdin, P.-O., Ed.), Academic Press, New York, 1966, p. 253. WFCss are the maxima of these localized orbitals denoting the highest negative charge concentration. For a review with some more background on Wannier functions and centers see: (d) Tse, J. S. Annu. Rev. Phys. Chem. 2002, 53, 249-290.

(15) (a) M. J. Frisch, G. W. Trucks, H. B. Schlegel, G. E. Scuseria, M. A. Robb, J. R. Cheeseman, J. A. Montgomery, Jr., T. Vreven, K. N. Kudin, J. C. Burant, J. M. Millam, S. S. Iyengar, J. Tomasi, V. Barone, B. Mennucci, M. Cossi, G. Scalmani, N. Rega, G. A. Petersson, H. Nakatsuji, M. Hada, M. Ehara, K. Toyota, R. Fukuda, J. Hasegawa, M. Ishida, T. Nakajima, Y. Honda, O. Kitao, H. Nakai, M. Klene, X. Li, J. E. Knox, H. P. Hratchian, J. B. Cross, C. Adamo, J. Jaramillo, R. Gomperts, R. E. Stratmann, O. Yazyev, A. J. Austin, R. Cammi, C. Pomelli, J. W. Ochterski, P. Y. Ayala, K. Morokuma, G. A. Voth, P. Salvador, J. J. Dannenberg, V. G. Zakrzewski, S. Dapprich, A. D. Daniels, M. C. Strain, O. Farkas, D. K. Malick, A. D. Rabuck, K. Raghavachari, J. B. Foresman, J. V. Ortiz, Q. Cui, A. G. Baboul, S. Clifford, J. Cioslowski, B. B. Stefanov, G. Liu, A. Liashenko, P. Piskorz, I. Komaromi, R. L. Martin, D. J. Fox, T. Keith, M. A. Al-Laham, C. Y. Peng, A. Nanayakkara, M. Challacombe, P. M. W. Gill, B. Johnson, W. Chen, M. W. Wong, C. Gonzalez, and J. A. Pople, Gaussian 03, Revision E.01, Gaussian, Inc., Pittsburgh PA, 2003; (b)M. J. Frisch, G. W. Trucks, H. B. Schlegel, G. E. Scuseria, M. A. Robb, J. R. Cheeseman, G. Scalmani, V. Barone, B. Mennucci, G. A. Petersson, H. Nakatsuji, M. Caricato, X. Li, H. P. Hratchian, A. F. Izmaylov, J. Bloino, G. Zheng, J. L. Sonnenberg, M. Hada, M. Ehara, K. Toyota, R. Fukuda, J. Hasegawa, M. Ishida, T. Nakajima, Y. Honda, O. Kitao, H. Nakai, T. Vreven, J. A. Montgomery, Jr., J. E. Peralta, F. Ogliaro, M. Bearpark, J. J. Heyd, E. Brothers, K. N. Kudin, V. N. Staroverov, R. Kobayashi, J. Normand, K. Raghavachari, A. Rendell, J. C. Burant, S. S. Iyengar, J. Tomasi, M. Cossi, N. Rega, J. M. Millam, M. Klene, J. E. Knox, J. B. Cross, V. Bakken, C. Adamo, J. Jaramillo, R. Gomperts, R. E. Stratmann, O. Yazyev, A. J. Austin, R. Cammi, C. Pomelli, J. W. Ochterski, R. L. Martin, K. Morokuma, V. G. Zakrzewski, G. A. Voth, P. Salvador, J. J. Dannenberg, S. Dapprich, A. D. Daniels, O. Farkas, J. B. Foresman, J. V. Ortiz, J. Cioslowski, and D. J. Fox, Gaussian 09, Revision A.02, Gaussian, Inc., Wallingford CT, 2009. 


\section{Cartesian coordinates of 1a and 2a and their water adducts}

\section{xyz format, B3LYP/SDD optimised}

$$
\begin{array}{rr}
13 & \\
1 \mathbf{a} & \text { (gas) } \\
\mathrm{U} & 0.000360 \\
\mathrm{O} & 0.420415 \\
\mathrm{O} & -0.159829 \\
\mathrm{O} & -2.193063 \\
\mathrm{O} & -1.667189 \\
\mathrm{O} & 1.964611 \\
\mathrm{O} & 2.397315 \\
\mathrm{O} & -1.263648 \\
\mathrm{H} & 2.093548 \\
\mathrm{H} & 2.419933 \\
\mathrm{H} & 2.639435 \\
\mathrm{H} & -2.060740 \\
\mathrm{H} & -1.114191
\end{array}
$$

$$
\begin{array}{rr}
-0.109678 & -0.004974 \\
-0.229290 & -1.774084 \\
0.306709 & 1.771761 \\
-0.216715 & -0.343090 \\
-1.517307 & -0.020266 \\
-1.203093 & 0.514618 \\
1.277379 & -0.265787 \\
2.299932 & 0.026087 \\
-1.395682 & 1.449362 \\
1.241774 & -1.229313 \\
0.367036 & 0.046293 \\
1.747836 & -0.059309 \\
2.388472 & 0.976634
\end{array}
$$

13

$\begin{array}{crrr}1 \mathrm{a}(\mathrm{PCM}) & & & \\ \mathrm{U} & -0.055039 & -0.080166 & -0.002928 \\ \mathrm{O} & 0.081577 & 0.030938 & -1.831336 \\ \mathrm{O} & 0.033100 & -0.134718 & 1.829376 \\ \mathrm{O} & -1.941196 & 1.128815 & 0.025400 \\ \mathrm{O} & -2.271246 & -0.290642 & -0.047247 \\ \mathrm{O} & 0.255799 & -2.278482 & -0.112856 \\ \mathrm{O} & 2.561703 & -0.135894 & 0.044094 \\ \mathrm{O} & 0.890286 & 2.310501 & 0.079628 \\ \mathrm{H} & 0.299259 & -2.838658 & 0.682568 \\ \mathrm{H} & 3.017984 & 0.435051 & -0.601626 \\ \mathrm{H} & 2.959328 & -1.022723 & -0.036186 \\ \mathrm{H} & 0.678061 & 3.016685 & -0.558207 \\ \mathrm{H} & 1.228767 & 2.740784 & 0.886329\end{array}$

$2 a(\mathrm{PCM})$

1.822235

$-1.760825$

$-0.180010$

0.151702

0.176594

$-0.144954$

$-0.258064$

$-0.539989$

$-0.084925$

$-1.068094$

0.514311

$-1.128228$
$-0.029037$

$-0.037943$

$-0.051033$

2.212720

$-0.591760$

$-2.582341$

$-0.444305$

$-0.713237$

$-3.152447$

$-2.825027$

$-0.402614$

2.729693
2. 374041
$-0.031595$

0.110359

$-0.140455$

0.720369

$-0.737976$

$-2.167571$

0.219523

2.156456

$-2.744030$

$-0.375074$

1. 134648

2. 734029

0.871514
0.012076

1. 832704

$-1.812651$

$-0.035044$

0.050696

0.179441

$-0.002100$

$-0.144666$

$-0.596695$

$-0.523698$

$-0.233852$

0.638318

$-0.942077$
16

1a.H2O (gas)

$\begin{array}{rr}\mathrm{U} & 0.212682 \\ \mathrm{O} & 0.971540 \\ \mathrm{O} & -0.447632 \\ \mathrm{O} & -1.763871 \\ \mathrm{O} & -0.923443 \\ \mathrm{O} & 2.218538 \\ \mathrm{O} & 2.238508 \\ \mathrm{O} & -1.297056 \\ \mathrm{H} & 2.211213 \\ \mathrm{H} & 2.480145 \\ \mathrm{H} & 2.620998 \\ \mathrm{H} & -1.531208 \\ \mathrm{H} & -2.097622 \\ \mathrm{O} & -3.207792 \\ \mathrm{H} & -2.947628 \\ \mathrm{H} & -2.613004\end{array}$

$-0.132260$

$-0.099886$

0.183138

$-0.909062$

$-1.984432$

$-0.760364$

1.758501

1. 882367

$-0.967258$

1. 775131

0.923012

1. 660953

1. 693540

0.736531

$-0.070751$

0.698960
$-0.028911$

$-1.686394$

1. 650626

$-0.746803$

$-0.281015$

0.910138

0.179479

$-0.753606$

1.850442

$-0.753610$

0.551473

$-1.662358$

$-0.204164$

0.844270

0.335826

1. 608661
-0.076391
-0.339881
0.335810
-1.055262
-1.962890
-0.942172
1.475983
1.769667
-1.112954
0.617997
1.482435
1.960944
-0.666150
1.012658
0.914270
1.480163
$-0.025640$

$-1.676824$

1. 632862

$-0.775348$

0.003486

0.927112

0.062848

$-0.718203$

1.874255

0.453115

$-0.863004$

$-1.660654$

$-0.143769$

0.677525

1. 520859

0.110376

$$
16
$$

1a.H2O (PCM)

$\begin{array}{rrrr}\mathrm{U} & 0.283843 & -0.102929 & -0.021054 \\ \mathrm{O} & 0.803350 & -0.085707 & -1.775044 \\ \mathrm{O} & -0.167450 & 0.086871 & 1.737937 \\ \mathrm{O} & -1.655402 & -1.161820 & -0.512908 \\ \mathrm{O} & -0.639192 & -2.130762 & -0.132486 \\ \mathrm{O} & 2.291032 & -0.891963 & 0.546894 \\ \mathrm{O} & 1.594001 & 2.151922 & 0.234392 \\ \mathrm{O} & -1.404863 & 1.802248 & -0.475518 \\ \mathrm{H} & 2.530950 & -1.039084 & 1.468981 \\ \mathrm{H} & 1.507097 & 2.857073 & -0.418615 \\ \mathrm{H} & 2.538066 & 2.045357 & 0.405504 \\ \mathrm{H} & -1.530605 & 2.124632 & -1.375636 \\ \mathrm{H} & -2.288833 & 1.515966 & -0.140293 \\ \mathrm{O} & -3.572869 & 0.454192 & 0.418119 \\ \mathrm{H} & -3.017444 & -0.354018 & 0.324092 \\ \mathrm{H} & -3.841613 & 0.519683 & 1.341882\end{array}$

16

2a.H2O (PCM)

$\begin{array}{lrrr}\mathrm{U} & -0.260868 & -0.074004 & 0.017825 \\ \mathrm{O} & -0.725718 & -0.347789 & 1.754204 \\ \mathrm{O} & 0.168003 & 0.274827 & -1.711572 \\ \mathrm{O} & 1.925930 & -1.132184 & 0.636072 \\ \mathrm{O} & 1.026947 & -2.015263 & -0.109982 \\ \mathrm{O} & -2.085691 & -1.177041 & -0.607003 \\ \mathrm{O} & -2.021594 & 1.791836 & -0.114954 \\ \mathrm{O} & 1.011570 & 1.757531 & 0.536420 \\ \mathrm{H} & -2.304969 & -1.305031 & -1.537390 \\ \mathrm{H} & -2.945834 & 1.523004 & -0.049567 \\ \mathrm{H} & -1.918712 & 2.607926 & 0.389242 \\ \mathrm{H} & 1.105336 & 2.021101 & 1.459293 \\ \mathrm{H} & 2.574233 & -0.761824 & -0.004319 \\ \mathrm{O} & 3.375069 & 0.875716 & -0.437318 \\ \mathrm{H} & 3.520297 & 1.138420 & -1.353271 \\ \mathrm{H} & 2.573384 & 1.363751 & -0.110851\end{array}$

